\title{
Synthesis and 3D-QSARs Analyses of Herbicidal $O, O$-Dialkyl-1-phenoxy- acetoxy-1-methylphosphonate Analogues as a New Class of Potent Inhibitors of Pyruvate Dehydrogenase
}

\author{
Min-Gyu Soung, Tae-Yeon Hwang, and Nack-Do Sung* \\ Department of Applied Biology and Chemistry, College of Agriculture and Life Science, Chungnam National University, \\ Daejeon 305-784, Korea. ${ }^{*}$ E-mail: ndsung15@hanmail.net \\ Received October 22, 2009, Accepted February 25, 2010
}

\begin{abstract}
A series of $O, O$-dialkyl-1-phenoxyacetoxy-1-methylphosphonate analogues (1 22) as a new class of potent inhibitors of pyruvate dehydrogenase were synthesized and 3D-QSARs (three dimensional qantitative structure-activity relationships) models on the pre-emergency herbicidal activity against the seed of cucumber (Cucumus Sativa L.) were derived and discussed quantitatively using comparative molecular field analysis (CoMFA) and comparative molecular similarity indeces analysis (CoMSIA) methods. The statistical values of CoMSIA models were better predictability and fitness than those of CoMFA models. The inhibitory activities according to the optimized CoMSIA model I were dependent on the electrostatic field (41.4\%), the H-bond acceptor field (26.0\%), the hydrophobic field (20.8\%) and the steric field $(11.7 \%)$. And also, it was found that the optimized CoMSIA model I with the sensitivity to the perturbation $\left(\mathrm{d}_{q}^{2} / \mathrm{dr}^{2} \mathrm{yy}^{\prime}=0.830\right)$ and the prediction $\left(\mathrm{q}^{2}=0.503\right)$ produced by a progressive scrambling analyses were not dependent on chance correlation. From the results of graphical analyses on the contour maps with the optimized CoMSIA model I, it is expected that the structural distinctions and descriptors that subscribe to herbicidal activities will be able to apply new an herbicide design.
\end{abstract}

Key Words: CoMSIA \& CoMFA analysis, Cucumber (Cucumus Sativa L.), O,O-Dialkyl-1-phenoxyacetoxy-1methylphosphonates, Herbicidal activity, Synthesis

\section{Introduction}

Proline $e^{1-4}$ is one of the known amino acids necessary for a plant's osmotic pressure during the germination, the pollen tube's germination as well as the flower's growth. The study ${ }^{5}$ on aminomethylene bisphosphonic acids that inhibits pyrroline-5carboxylate reductase ( $\mathrm{P} 5 \mathrm{C}: \mathrm{EC}$ 1.5.1.2), which is known as the catalyst to the last stage of proline biosynthesis necessary for the integration of such proteins and cells walls, is in progress. Also, imidazoleglycerolphosphate dehydratase (IGPD:EC 4.2. 1.19), which affects the sixth level of the histidine biosynthesis pathway and is known to play an important role in the development of herbicides, produces ${ }^{6}$ imidazoleacetolphospate (IAP) through the hydration of imidazoleglycerolphospate (IGP). The mechanism of such enzymes has not yet been fully identified. Still, researches ${ }^{7}$ on the phloem mobile herbicides using triazolephosphonate derivatives that progress through IGPD's diazafulvene intermediate are being performed. Most importantly, the pyruvate dehydrogenase complex (PDHc) is already known to be the center of the representative inhibition reaction. ${ }^{8}$ The $\mathrm{PDHc}$ is constituted a total of three types of enzymes and one cofactor. ${ }^{9}$ The phosphonate compounds undergo competitive reactions with the pyruvate in the plant body and show the inhibitory activity against the selective pyruvate dehydrogenase (PDH) ${ }^{10}$ Specifically, the PDH enzyme is produced AcetylCoA from the pyruvate through the thiamine pyrophosphate (TPP) enzyme by the oxidative decarboxylation. ${ }^{11-12}$ Among those, PDHc E1 component (E.C. 1.2.4.1) is the constituent structure that first affects the production of Acetyl-CoA using PDHc's TPP enzyme and cofactor's $\mathrm{Mg}^{2+}$ metal as opposed to the other various procedures. ${ }^{13,14}$ Therefore PDHc Elenzyme is a target of the pesticide design and the research was planned for the purpose of inhibiting this enzyme. ${ }^{15}$

For the past few years, it was observed that phenoxyacetoxyalkylphosphonates derivatives showed high herbicidal activity as a PDH inhibitor. ${ }^{16,17}$ Also, the phosphonate monosalt has a structure that resembles the pyruvate, which is a substrate of the PDHc, and is reported to show higher herbicidal effect. ${ }^{18}$ In this study, a series of $O, O$-dialkyl-1-phenoxyacetoxy-1methylphosphonate analogues as substrate molecules (1 22) were synthesized and the herbicidal activity ${ }^{19,20}$ by a change in the substituents $\left(\mathrm{R}_{1}-\mathrm{R}_{4}\right)$ against the seed of cucumber (Cucumis sativus L.) in the pre-emergence step was measured. Based upon those findings, the three dimensional quantitative structureactivity relationships (3D-QSAR) ${ }^{21}$ results on the herbicidal effect of several compounds predicted ${ }^{22}$ by the authors were discussed quantitatively.

\section{Materials and Methods}

Reagents and general. All commercial reagents and solvents were used without further purification unless otherwise specified. Solvents and reagents were purchased from Sigma-Aldrich and Fluka. Thin layer chromatography (TLC) was performed on Merck 60 F-254 silica plates and visualized by UV. Flash column chromatography was performed on silica gel (Merck, 230 - 400 mesh). ${ }^{1} \mathrm{H}$ NMR spectra were obtained using Inova 600 model $(600 \mathrm{MHz})$ under the conditions of adding TMS as an internal standard material to the $\mathrm{CDCl}_{3}$ solvent. Mass spectra were obtained using API $3000+1100$ series LC mass spectro- 
meter.

General synthetic procedure for substrate molecules (1 22). A solution of $\mathrm{R}_{1}, \mathrm{R}_{2}$-phenoxyacetyl chloride ${ }^{18,20}(5.2 \mathrm{mM})$ in trichloromethane $(10 \mathrm{~mL})$ was added dropwise to stirred mixture of $O, O-1-\mathrm{R}_{4}$-hydroxymethyldimethylphosphate ${ }^{18,20}(5 \mathrm{mM})$ and triethylamine $(0.53 \mathrm{~g}, 5.0 \mathrm{mM})$ in trichloromethane $(15 \mathrm{~mL})$ at $0{ }^{\circ} \mathrm{C}$. The reaction mixture was stirred at an ambient temperature for 3 hours, washed with hydrochloric acid ( $0.1 \mathrm{M}$ solution, $25 \mathrm{~mL}$ ) and brine (25 mL) separately; dried; and evaporated. The residue was chromatographed on silica with ethylacetate and $n$-hexane solution to give $O, O-\mathrm{R}_{3}, \mathrm{R}_{3}-1-\left(\mathrm{R}_{1}, \mathrm{R}_{2}\right.$-phenoxyacetoxy)-1-( $\left(\mathrm{R}_{4}\right)$-methylphosphonates $(\mathbf{1} \sim \mathbf{2 2})$ as a yellow liquid.

$\boldsymbol{O}, O$-Dimethyl-1-(2-fluoro-4-chlorophenoxyacetoxy)-1-(2chlorophenyl)methylphosphonate (1): yellow liquid; $R_{f}=0.68$ (EtOAc $: n$-Hex $=4: 1) ;{ }^{1} \mathrm{H} \mathrm{NMR}\left(\mathrm{CDCl}_{3} / \mathrm{TMS}\right) \delta 3.64\left(\mathrm{~d}, J_{\mathrm{HP}}=\right.$ $\left.10.8 \mathrm{~Hz}, 3 \mathrm{H}, \mathrm{OCH}_{3}\right), 3.79\left(\mathrm{~d}, J_{\mathrm{HP}}=10.8 \mathrm{~Hz}, 3 \mathrm{H}, \mathrm{OCH}_{3}\right), 4.75-$ $4.83\left(\mathrm{~m}, 2 \mathrm{H}, \mathrm{OCH}_{2} \mathrm{CO}\right), 6.73-6.75\left(\mathrm{~d}, J_{\mathrm{HP}}=13.2 \mathrm{~Hz}, 1 \mathrm{H}, \mathrm{OCHP}\right)$, 6.83-7.53 (m, 7H, phenyl-H); ESI-MS m/z (\%) $459[\mathrm{M}+\mathrm{Na}]^{+}$ (100), 233 (25), 155 (8), 124 (3).

$O, O$-Dimethyl-1-(2-chloro-4-bromophenoxyacetoxy)1-(2-chlorophenyl)methylphosphonate (2): yellow liquid; $R_{f}=$ $0.68($ EtOAc $: n$-Hex $=4: 1) ;{ }^{1} \mathrm{H} \mathrm{NMR}\left(\mathrm{CDCl}_{3} / \mathrm{TMS}\right) \delta 3.65(\mathrm{~d}$, $\left.J_{\mathrm{HP}}=10.8 \mathrm{~Hz}, 3 \mathrm{H}, \mathrm{OCH}_{3}\right), 3.79\left(\mathrm{~d}, J_{\mathrm{HP}}=10.8 \mathrm{~Hz}, 3 \mathrm{H}, \mathrm{OCH}_{3}\right)$, 4.77-4.84 (m, 2H, $\left.\mathrm{OCH}_{2} \mathrm{CO}\right), 6.68-7.53(\mathrm{~m}, 8 \mathrm{H}, \mathrm{OCHP}$ and phenyl-H); ESI-MS m/z (\%) 521 [M+Na] ${ }^{+}$(82), 499 (25), 273 (14), 233 (100), 194 (10), 159 (15), 141 (38), 117 (39).

$O, O$-Dimethyl-1-(3,4-dichlorophenoxyacetoxy)-1-(2-chlorophenyl)methylphosphonate (3): yellow liquid; $R_{f}=0.63$ (EtOAc : $n$-Hex $=4: 1) ;{ }^{1} \mathrm{H} \mathrm{NMR}\left(\mathrm{CDCl}_{3} / \mathrm{TMS}\right) \delta 3.64\left(\mathrm{~d}, J_{\mathrm{HP}}=\right.$ $\left.10.8 \mathrm{~Hz}, 3 \mathrm{H}, \mathrm{OCH}_{3}\right), 3.79$ (d, $\left.J_{\mathrm{HP}}=10.8 \mathrm{~Hz}, 3 \mathrm{H}, \mathrm{OCH}_{3}\right), 4.68-$ 4.77 (m, 2H, $\left.\mathrm{OCH}_{2} \mathrm{CO}\right), 6.74-7.53$ (m, 8H, OCHP and phenyl$\mathrm{H})$; ESI-MS $m / z(\%) 475[\mathrm{M}+\mathrm{Na}]^{+}$(89), 455 (22), 273 (48), 233 (100), 198 (12), 155 (24), 141 (66).

$O, O$-Dimethyl-1-(3-fluoro-4-chlorophenoxyacetoxy)-1-(2chlorophenyl)methylphosphonate (4): yellow liquid; $R_{f}=0.68$ (EtOAc : $n$-Hex $=4: 1) ;{ }^{1} \mathrm{H} \mathrm{NMR}\left(\mathrm{CDCl}_{3} / \mathrm{TMS}\right): \delta 3.64\left(\mathrm{~d}, J_{\mathrm{HP}}=\right.$ $\left.10.8 \mathrm{~Hz}, 3 \mathrm{H}, \mathrm{OCH}_{3}\right), 3.79-3.80\left(\mathrm{~d}, J_{\mathrm{HP}}=10.8 \mathrm{~Hz}, 3 \mathrm{H}, \mathrm{OCH}_{3}\right)$, 4.68-4.77 (m, 2H, $\left.\mathrm{OCH}_{2} \mathrm{CO}\right), 6.61-7.54(\mathrm{~m}, 8 \mathrm{H}, \mathrm{OCHP}$ and phenyl-H); ESI-MS m/z (\%) 441 (10), 439 (65), 438 (15), 437 $[\mathrm{M}]^{+}$(100), 251 (10), 235 (17), 234 (5), 233 (48).

$O, O$-Dimethyl-1-(4-cyanophenoxyacetoxy)-1-(2-chlorophenyl)methylphosphonate (5): yellow liquid; $R_{f}=0.55$ (EtOAc: $n$-Hex $=4: 1) ;{ }^{1} \mathrm{H} \mathrm{NMR}\left(\mathrm{CDCl}_{3} / \mathrm{TMS}\right): \delta 3.62\left(\mathrm{~d}, J_{\mathrm{HP}}=10.8 \mathrm{~Hz}\right.$, $\left.3 \mathrm{H}, \mathrm{OCH}_{3}\right), 3.80\left(\mathrm{~d}, J_{\mathrm{HP}}=10.8 \mathrm{~Hz}, 3 \mathrm{H}, \mathrm{OCH}_{3}\right), 4.76-4.85(\mathrm{~m}$, $\left.2 \mathrm{H}, \mathrm{OCH}_{2} \mathrm{CO}\right), 6.74-7.59$ (m, 9H, OCHP and phenyl-H); ESI$\mathrm{MS} m / z(\%) 432[\mathrm{M}+\mathrm{Na}]^{+}$(100), 410 (12), 233 (74), 198 (8), 157 (8), 155 (21), 141 (66).

$O, O$-Dimethyl-1-(3-cyanophenoxyacetoxy)-1-(2-chlorophenyl)methylphosphonate (6): yellow liquid; $R_{f}=0.58$ (EtOAc: $n$-Hex $=4: 1) ;{ }^{1} \mathrm{H} \mathrm{NMR}\left(\mathrm{CDCl}_{3} / \mathrm{TMS}\right): \delta 3.64\left(\mathrm{~d}, J_{\mathrm{HP}}=10.8 \mathrm{~Hz}\right.$, $\left.3 \mathrm{H}, \mathrm{OCH}_{3}\right), 3.80\left(\mathrm{~d}, J_{\mathrm{HP}}=10.8 \mathrm{~Hz}, 3 \mathrm{H}, \mathrm{OCH}_{3}\right), 4.74-4.83(\mathrm{~m}$, $\left.2 \mathrm{H}, \mathrm{OCH}_{2} \mathrm{CO}\right), 6.74-7.56$ (m, 9H, OCHP and phenyl-H); ESIMS $m / z(\%) 432[\mathrm{M}+\mathrm{Na}]^{+}$(100), $410(21), 331$ (4), 273 (5), 233 (74), 198 (4), 155 (14), 141 (19), 117 (12).

$\boldsymbol{O}, \boldsymbol{O}$-Dimethyl-1-(4-cyanomethylphenoxyacetoxy)-1-(2chlorophenyl)methylphosphonate (7): yellow liquid; $R_{f}=0.62$ (EtOAc $: n$-Hex $=4: 1) ;{ }^{1} \mathrm{H} \mathrm{NMR}\left(\mathrm{CDCl}_{3} / \mathrm{TMS}\right) \delta 3.64\left(\mathrm{~d}, J_{\mathrm{HP}}=\right.$ $\left.10.8 \mathrm{~Hz}, 3 \mathrm{H}, \mathrm{OCH}_{3}\right), 3.68$ (s, $\left.2 \mathrm{H}, \mathrm{CH}_{2} \mathrm{CN}\right), 3.79$ (d, $J_{\mathrm{HP}}=10.8$
$\left.\mathrm{Hz}, 3 \mathrm{H}, \mathrm{OCH}_{3}\right), 4.70-4.80$ (m, 2H, $\left.\mathrm{OCH}_{2} \mathrm{CO}\right), 6.74-7.55$ (m, 9H, OCHP and phenyl-H); ESI-MS $m / z(\%) 446[\mathrm{M}+\mathrm{Na}]^{+}(100), 424$ (38), 411 (6), 247 (6), 233 (82), 198 (6), 158 (6), 155 (18), 141 (32), 124 (36).

$O, O$-Dimethyl-1-(4-mothoxyphenoxyacetoxy)-1-(2chlorophenyl)methylphosphonate (8): yellow liquid; $R_{f}=$ 0.67 (EtOAc : $n$-Hex $=4: 1) ;{ }^{1} \mathrm{H} \mathrm{NMR}\left(\mathrm{CDCl}_{3} / \mathrm{TMS}\right) \delta 3.65(\mathrm{~d}$, $\left.J_{\mathrm{HP}}=10.8 \mathrm{~Hz}, 3 \mathrm{H}, \mathrm{OCH}_{3}\right), 3.66\left(\mathrm{~d}, J_{\mathrm{HP}}=10.8 \mathrm{~Hz}, 3 \mathrm{H}, \mathrm{OCH}_{3}\right)$, $3.76\left(\mathrm{~s}, 3 \mathrm{H}, \mathrm{OCH}_{3}\right), 4.65-4.74\left(\mathrm{~m}, 2 \mathrm{H}, \mathrm{OCH}_{2} \mathrm{CO}\right), 6.74-7.54$ (m, 9H, OCHP and phenyl-H); ESI-MS m/z (\%) 437 [M+Na] (100), 415 (34), 233 (20), 155 (8), 141 (13).

$O, O$-Dimethyl-1-(3-mothoxyphenoxyacetoxy)-1-(2chlorophenyl)methylphosphonate (9): yellow liquid; $R_{f}=$ 0.69 (EtOAc : $n$-Hex $=4: 1) ;{ }^{1} \mathrm{H} \mathrm{NMR}\left(\mathrm{CDCl}_{3} / \mathrm{TMS}\right) \delta 3.64(\mathrm{~d}$, $\left.J_{\mathrm{HP}}=10.8 \mathrm{~Hz}, 3 \mathrm{H}, \mathrm{OCH}_{3}\right), 3.76\left(\mathrm{~d}, J_{\mathrm{HP}}=10.8 \mathrm{~Hz}, 3 \mathrm{H}, \mathrm{OCH}_{3}\right)$, $3.78\left(\mathrm{~s}, 3 \mathrm{H}, \mathrm{OCH}_{3}\right), 4.68-4.80$ (m, $\left.2 \mathrm{H}, \mathrm{OCH}_{2} \mathrm{CO}\right), 6.44-7.54$ (m, 9H, OCHP and phenyl-H); ESI-MS m/z (\%) $437[\mathrm{M}+\mathrm{Na}]^{+}$ (100), 415 (31), 305 (22), 273 (25), 233 (36), 198 (6), 163 (9), 155 (13), 141 (42).

$O, O$-Dimethyl-1-(4-propylphenoxyacetoxy)-1-(2-chlorophenyl)methylphosphonate (10): yellow liquid; $R_{f}=0.76$ (EtOAc : $n$-Hex $=4: 1) ;{ }^{1} \mathrm{H} \mathrm{NMR}\left(\mathrm{CDCl}_{3} / \mathrm{TMS}\right) \delta 0.91\left(\mathrm{t}, J_{\mathrm{HH}}=\right.$ $\left.3.0 \mathrm{~Hz}, 3 \mathrm{H}, \mathrm{CH}_{2} \mathrm{CH}_{2} \mathrm{CH}_{3}\right)$, 1.56-1.61 (m, $2 \mathrm{H}, \mathrm{CH}_{2} \mathrm{CH}_{2} \mathrm{CH}_{3}$ ), $2.51\left(\mathrm{t}, J_{\mathrm{HH}}=7.2 \mathrm{~Hz}, 2 \mathrm{H}, \mathrm{CH}_{2} \mathrm{CH}_{2} \mathrm{CH}_{3}\right), 3.64\left(\mathrm{~d}, J_{\mathrm{HP}}=10.8 \mathrm{~Hz}\right.$, $\left.3 \mathrm{H}, \mathrm{OCH}_{3}\right), 3.78\left(\mathrm{~d}, \mathrm{~J}_{\mathrm{HP}}=10.8 \mathrm{~Hz}, 3 \mathrm{H}, \mathrm{OCH}_{3}\right), 4.63-4.76(\mathrm{~m}$, $\left.2 \mathrm{H}, \mathrm{OCH}_{2} \mathrm{CO}\right), 6.74-7.53$ (m, 9H, OCHP and phenyl-H); ESIMS $m / z(\%) 449[\mathrm{M}+\mathrm{Na}]^{+}$(100), 427 (31), 233 (19), 155 (4), 100 (4).

$O, O$-Dimethyl-1-(4-isopropylphenoxyaceto $x y)-1-(2-$ chlorophenyl)methylphosphonate (11): yellow liquid; $R_{f}=0.78$ (EtOAc : $n$-Hex $=4: 1) ;{ }^{1} \mathrm{H} \mathrm{NMR}\left(\mathrm{CDCl}_{3} / \mathrm{TMS}\right) \delta 1.21\left(\mathrm{~d}, J_{\mathrm{HH}}=\right.$ $\left.7.2 \mathrm{~Hz}, 6 \mathrm{H}, \mathrm{CH}\left(\mathrm{CH}_{3}\right)_{2}\right), 2.85\left(\mathrm{~m}, J_{\mathrm{HH}}=7.2 \mathrm{~Hz}, 1 \mathrm{H}, \mathrm{CH}\left(\mathrm{CH}_{3}\right)_{2}\right)$, $3.64\left(\mathrm{~d}, J_{\mathrm{HP}}=10.8 \mathrm{~Hz}, 3 \mathrm{H}, \mathrm{OCH}_{3}\right), 3.77\left(\mathrm{~d}, J_{\mathrm{HP}}=10.8 \mathrm{~Hz}, 3 \mathrm{H}\right.$, $\left.\mathrm{OCH}_{3}\right), 4.63-4.77$ (m, 2H, OCH$\left.{ }_{2} \mathrm{CO}\right), 6.74-7.53$ (m, 9H, OCHP and phenyl-H); ESI-MS m/z (\%) $429(35), 428(18), 427[\mathrm{M}+\mathrm{H}]^{+}$ (100), 250 (14), 233(15).

$O, O$-Dimethyl-1-(4-ethylphenoxyacetoxy)-1-(2-chlorophenyl)methylphosphonate (12): yellow liquid; $R_{f}=0.73$ (EtOAc $: n$-Hex $=4: 1) ;{ }^{1} \mathrm{H} \mathrm{NMR}\left(\mathrm{CDCl}_{3} / \mathrm{TMS}\right) \delta 1.19\left(\mathrm{t}, J_{\mathrm{HH}}=7.2\right.$ $\mathrm{Hz}, 3 \mathrm{H}, \mathrm{CH}_{2} \mathrm{CH}_{3}$ ), 2.58 (q, $\left.J_{\mathrm{HH}}=7.2 \mathrm{~Hz}, 2 \mathrm{H}, \mathrm{CH}_{2} \mathrm{CH}_{3}\right), 3.64$ (d, $\left.J_{\mathrm{HP}}=10.8 \mathrm{~Hz}, 3 \mathrm{H}, \mathrm{OCH}_{3}\right), 3.78\left(\mathrm{~d}, J_{\mathrm{HP}}=10.8 \mathrm{~Hz}, 3 \mathrm{H}, \mathrm{OCH}_{3}\right)$, 4.67-4.77 (m, 2H, $\left.\mathrm{OCH}_{2} \mathrm{CO}\right), 6.74-7.54$ (m, 9H, OCHP and phenyl-H); ESI-MS m/z (\%) 415 (33), 414 (20), $413[\mathrm{M}+\mathrm{H}]^{+}$ (100), 235 (5), 233(15).

$\boldsymbol{O}, \boldsymbol{O}$-Dimethyl-1-(2-fluoro-4-chlorophenoxyacetoxy)ethylphosphonate (13): yellow liquid; $R_{f}=0.48$ (EtOAc : $n-\mathrm{Hex}=$ $4: 1) ;{ }^{1} \mathrm{H} \mathrm{NMR}\left(\mathrm{CDCl}_{3} / \mathrm{TMS}\right) \delta 1.50\left(\mathrm{~d}, J_{\mathrm{HH}}=7.2 \mathrm{~Hz}, 3 / 2 \mathrm{H}, \mathrm{PCH}-\right.$ $\left.\mathrm{CH}_{3}\right), 1.52\left(\mathrm{~d}, J_{\mathrm{HH}}=7.2 \mathrm{~Hz}, 3 / 2 \mathrm{H}, \mathrm{PCHCH}_{3}\right), 3.78\left(\mathrm{~d}, J_{\mathrm{HP}}=10.8\right.$ $\left.\mathrm{Hz}, 3 \mathrm{H}, \mathrm{OCH}_{3}\right), 3.81\left(\mathrm{~d}, \mathrm{~J}_{\mathrm{HP}}=10.8 \mathrm{~Hz}, 3 \mathrm{H}, \mathrm{OCH}_{3}\right), 4.73(\mathrm{~s}, 2 \mathrm{H}$, $\mathrm{OCH}_{2} \mathrm{CO}$ ), 5.39 (q, $\left.J_{\mathrm{HH}}=7.2 \mathrm{~Hz}, 1 \mathrm{H}, \mathrm{OCHP}\right), 6.87-7.14(\mathrm{~m}, 3 \mathrm{H}$, phenyl-H); ESI-MS m/z (\%) 343 (38), 342 (10), $341[\mathrm{M}+\mathrm{H}]^{+}$ (100), 235 (5), 233(15).

O,O-Dimethyl-1-(2-chloro-4-bromophenoxyacetoxy)ethylphosphonate (14): yellow liquid; $R_{f}=0.52$ (EtOAc : $n$-Hex $=$ $4: 1) ;{ }^{1} \mathrm{H} \mathrm{NMR}\left(\mathrm{CDCl}_{3} / \mathrm{TMS}\right) \delta 1.50\left(\mathrm{~d}, J_{\mathrm{HH}}=7.2 \mathrm{~Hz}, 3 / 2 \mathrm{H}, \mathrm{PCH}-\right.$ $\left.\mathrm{CH}_{3}\right), 1.52\left(\mathrm{~d}, J_{\mathrm{HH}}=7.2 \mathrm{~Hz}, 3 / 2 \mathrm{H}, \mathrm{PCHCH}_{3}\right), 3.77\left(\mathrm{~d}, J_{\mathrm{HP}}=10.8\right.$ $\left.\mathrm{Hz}, 3 \mathrm{H}, \mathrm{OCH}_{3}\right), 3.79\left(\mathrm{~d}, J_{\mathrm{HP}}=10.8 \mathrm{~Hz}, 3 \mathrm{H}, \mathrm{OCH}_{3}\right), 4.75(\mathrm{~s}, 2 \mathrm{H}$, $\mathrm{OCH}_{2} \mathrm{CO}$ ), 5.39 (q, $\left.J_{\mathrm{HH}}=7.2 \mathrm{~Hz}, 1 \mathrm{H}, \mathrm{OCHP}\right), 6.73-7.53$ (m, $3 \mathrm{H}$, 
phenyl-H); ESI-MS m/z (\%) 404 (23), 403 (10), $402[\mathrm{M}+\mathrm{H}]^{+}$ (100), 401 (72).

$O, O$-Dimethyl-1-(3,4-dichlorophenoxyacetoxy)ethylphosphonate (15): yellow liquid; $R_{f}=0.52$ (EtOAc : $n$-Hex $=$ $4: 1) ;{ }^{1} \mathrm{H} \mathrm{NMR}\left(\mathrm{CDCl}_{3} / \mathrm{TMS}\right) \delta 1.50\left(\mathrm{~d}, J_{\mathrm{HH}}=7.2 \mathrm{~Hz}, 3 / 2 \mathrm{H}\right.$, $\left.\mathrm{PCHCH}_{3}\right), 1.53\left(\mathrm{~d}, J_{\mathrm{HH}}=7.2 \mathrm{~Hz}, 3 / 2 \mathrm{H}, \mathrm{PCHCH}_{3}\right), 3.78\left(\mathrm{~d}, J_{\mathrm{HP}}=\right.$ $\left.10.8 \mathrm{~Hz}, 3 \mathrm{H}, \mathrm{OCH}_{3}\right), 3.80\left(\mathrm{~d}, J_{\mathrm{HP}}=10.8 \mathrm{~Hz}, 3 \mathrm{H}, \mathrm{OCH}_{3}\right), 4.67$ (s, $\left.2 \mathrm{H}, \mathrm{OCH}_{2} \mathrm{CO}\right), 5.40$ (q, $\left.J_{\mathrm{HH}}=7.2 \mathrm{~Hz}, 1 \mathrm{H}, \mathrm{OCHP}\right), 6.77-$ 7.35 (m, 3H, phenyl-H); ESI-MS m/z (\%) 359 (67), 358 (12), $357[\mathrm{M}]^{+}$(100).

$O, O$-Dimethyl-1-(3-fluoro-4-chlorophenoxyacetoxy)ethylphosphonate (16): yellow liquid; $R_{f}=0.52$ (EtOAc : $n$-Hex $=$ $4: 1) ;{ }^{1} \mathrm{H} \mathrm{NMR}\left(\mathrm{CDCl}_{3} / \mathrm{TMS}\right) \delta 1.50\left(\mathrm{~d}, J_{\mathrm{HH}}=7.2 \mathrm{~Hz}, 3 / 2 \mathrm{H}\right.$, $\left.\mathrm{PCHCH}_{3}\right), 1.53\left(\mathrm{~d}, J_{\mathrm{HH}}=7.2 \mathrm{~Hz}, 3 / 2 \mathrm{H}, \mathrm{PCHCH}_{3}\right), 3.77\left(\mathrm{~d}, J_{\mathrm{HP}}=\right.$ $\left.10.8 \mathrm{~Hz}, 3 \mathrm{H}, \mathrm{OCH}_{3}\right), 3.80\left(\mathrm{~d}, J_{\mathrm{HP}}=10.8 \mathrm{~Hz}, 3 \mathrm{H}, \mathrm{OCH}_{3}\right), 4.65(\mathrm{~s}$, $\left.2 \mathrm{H}, \mathrm{OCH}_{2} \mathrm{CO}\right), 5.40$ (q, $\left.J_{\mathrm{HH}}=7.2 \mathrm{~Hz}, 1 \mathrm{H}, \mathrm{OCHP}\right), 6.65-7.31(\mathrm{~m}$, $3 \mathrm{H}$, phenyl-H); ESI-MS m/z (\%) 343 (35), 342 (14), $341[\mathrm{M}+\mathrm{H}]^{+}$ (100), 262 (5), 260 (17).

$\boldsymbol{O}, \boldsymbol{O}$-Dimethyl-1-(4-cyanophenoxyacetoxy)ethylphosphonate (17): yellow liquid; $R_{f}=0.55$ (EtOAc : $n$-Hex $\left.=4: 1\right) ;{ }^{1} \mathrm{H}$ $\mathrm{NMR}\left(\mathrm{CDCl}_{3} / \mathrm{TMS}\right) \delta 1.50\left(\mathrm{~d}, J_{\mathrm{HH}}=7.2 \mathrm{~Hz}, 3 / 2 \mathrm{H}, \mathrm{PCHCH}_{3}\right)$, $1.53\left(\mathrm{~d}, J_{\mathrm{HH}}=7.2 \mathrm{~Hz}, 3 / 2 \mathrm{H}, \mathrm{PCHCH}_{3}\right), 3.76\left(\mathrm{~d}, J_{\mathrm{HP}}=10.8 \mathrm{~Hz}\right.$, $\left.3 \mathrm{H}, \mathrm{OCH}_{3}\right), 3.78\left(\mathrm{~d}, J_{\mathrm{HP}}=10.8 \mathrm{~Hz}, 3 \mathrm{H}, \mathrm{OCH}_{3}\right), 4.66(\mathrm{~s}, 2 \mathrm{H}$, $\mathrm{OCH}_{2} \mathrm{CO}$ ), 5.41 (q, $\left.J_{\mathrm{HH}}=7.2 \mathrm{~Hz}, 1 \mathrm{H}, \mathrm{OCHP}\right), 6.82\left(\mathrm{~d}, J_{\mathrm{HH}}=7.8\right.$ $\mathrm{Hz}, 2 \mathrm{H}$, phenyl-H), 7.09 (d, $J_{\mathrm{HH}}=7.8 \mathrm{~Hz}, 2 \mathrm{H}$, phenyl-H); ESIMS $m / z(\%) 336[\mathrm{M}+\mathrm{Na}]^{+}(10), 315(14), 314[\mathrm{M}+\mathrm{H}]^{+}(100)$.

$\boldsymbol{O}, \boldsymbol{O}$-Dimethyl-1-(3-cyanophenoxyacetoxy)ethylphosphonate (18): yellow liquid; $R_{f}=0.45$ (EtOAc : $n$-Hex $\left.=4: 1\right) ;{ }^{1} \mathrm{H}$ $\mathrm{NMR}\left(\mathrm{CDCl}_{3} / \mathrm{TMS}\right) \delta 1.51\left(\mathrm{~d}, J_{\mathrm{HH}}=7.2 \mathrm{~Hz}, 3 / 2 \mathrm{H}, \mathrm{PCHCH}_{3}\right)$, $1.52\left(\mathrm{~d}, J_{\mathrm{HH}}=7.2 \mathrm{~Hz}, 3 / 2 \mathrm{H}, \mathrm{PCHCH}_{3}\right), 3.78\left(\mathrm{~d}, J_{\mathrm{HP}}=10.8 \mathrm{~Hz}\right.$, $\left.3 \mathrm{H}, \mathrm{OCH}_{3}\right), 3.81\left(\mathrm{~d}, J_{\mathrm{HP}}=10.8 \mathrm{~Hz}, 3 \mathrm{H}, \mathrm{OCH}_{3}\right), 4.72(\mathrm{~s}, 2 \mathrm{H}$, $\mathrm{OCH}_{2} \mathrm{CO}$ ), 5.40 (q, $\left.J_{\mathrm{HH}}=7.2 \mathrm{~Hz}, 1 \mathrm{H}, \mathrm{OCHP}\right), 7.13-7.42(\mathrm{~m}, 4 \mathrm{H}$, phenyl-H); ESI-MS m/z (\%) 336 [M+Na] ${ }^{+}$(16), 315 (14), 314 $[\mathrm{M}+\mathrm{H}]^{+}(100)$.

$O, O$-Dimethyl-1-(4-cyanomethylphenoxyacetoxy)ethylphosphonate (19): yellow liquid; $R_{f}=0.43$ (EtOAc : $n$-Hex $=$ $4: 1) ;{ }^{1} \mathrm{H} \mathrm{NMR}\left(\mathrm{CDCl}_{3} / \mathrm{TMS}\right) \delta 1.50\left(\mathrm{~d}, J_{\mathrm{HH}}=7.2 \mathrm{~Hz}, 3 / 2 \mathrm{H}\right.$, $\left.\mathrm{PCHCH}_{3}\right), 1.53\left(\mathrm{~d}, J_{\mathrm{HH}}=7.2 \mathrm{~Hz}, 3 / 2 \mathrm{H}, \mathrm{PCHCH}_{3}\right), 3.69(\mathrm{~s}, 2 \mathrm{H}$, $\left.\mathrm{CH}_{2} \mathrm{CN}\right), 3.78\left(\mathrm{~d}, J_{\mathrm{HP}}=10.8 \mathrm{~Hz}, 3 \mathrm{H}, \mathrm{OCH}_{3}\right), 3.81\left(\mathrm{~d}, J_{\mathrm{HP}}=\right.$ $\left.10.8 \mathrm{~Hz}, 3 \mathrm{H}, \mathrm{OCH}_{3}\right), 4.69$ (s, $2 \mathrm{H}, \mathrm{OCH}_{2} \mathrm{CO}$ ), 5.40 (q, $J_{\mathrm{HH}}=7.2$ $\mathrm{Hz}, 1 \mathrm{H}, \mathrm{OCHP}), 6.90-7.27$ (m, 4H, phenyl-H); ESI-MS m/z (\%) $377[2 \mathrm{M}+\mathrm{Na}]^{+}(5), 656(14), 655[2 \mathrm{M}+\mathrm{Na}]^{+}(53), 350[\mathrm{M}+\mathrm{Na}]^{+}$ (8), $329(15), 328[\mathrm{M}+\mathrm{H}]^{+}(100)$.

$O, O$-Dimethyl-1-(4-mothoxyphenoxyacetoxy)ethylphosphonate (20): yellow liquid; $R_{f}=0.44$ (EtOAc : $n$-Hex $=$ 4:1); ${ }^{1} \mathrm{H}$ NMR $\left(\mathrm{CDCl}_{3} / \mathrm{TMS}\right) \delta 1.50\left(\mathrm{~d}, J_{\mathrm{HH}}=7.2 \mathrm{~Hz}, 3 / 2 \mathrm{H}\right.$, $\left.\mathrm{PCHCH}_{3}\right), 1.53\left(\mathrm{~d}, J_{\mathrm{HH}}=7.2 \mathrm{~Hz}, 3 / 2 \mathrm{H}, \mathrm{PCHCH}_{3}\right), 3.77\left(\mathrm{~d}, J_{\mathrm{HP}}=\right.$ $\left.10.8 \mathrm{~Hz}, 3 \mathrm{H}, \mathrm{OCH}_{3}\right), 3.78(\mathrm{~s}, 3 \mathrm{H}$, phenyl-OCH$), 3.79\left(\mathrm{~d}, J_{\mathrm{HP}}=\right.$ $\left.10.8 \mathrm{~Hz}, 3 \mathrm{H}, \mathrm{OCH}_{3}\right), 4.64$ (s, $\left.2 \mathrm{H}, \mathrm{OCH}_{2} \mathrm{CO}\right), 5.40$ (q, $J_{\mathrm{HH}}=7.2$ $\mathrm{Hz}, 1 \mathrm{H}, \mathrm{OCHP}), 6.82-7.26$ (m, 4H, phenyl-H); ESI-MS $m / z$ (\%) $659[2 \mathrm{M}+\mathrm{Na}]^{+}(5), 638(18), 637[2 \mathrm{M}+\mathrm{H}]^{+}(65), 600(10)$, $320(15), 319[\mathrm{M}+\mathrm{H}]^{+}(100)$.

$O, O$-Dimethyl-1-(4-is opropylpheno xyace to xy)ethylphosphonate (21): yellow liquid; $R_{f}=0.53$ (EtOAc : $n$-Hex $=$ $4: 1) ;{ }^{1} \mathrm{H}$ NMR $\left(\mathrm{CDCl}_{3} / \mathrm{TMS}\right) \delta 1.21\left(\mathrm{~d}, J_{\mathrm{HH}}=7.2 \mathrm{~Hz}, 6 \mathrm{H}\right.$, $\left.\mathrm{CH}\left(\mathrm{CH}_{3}\right)_{2}\right), 1.50\left(\mathrm{~d}, J_{\mathrm{HH}}=7.2 \mathrm{~Hz}, 3 / 2 \mathrm{H}, \mathrm{PCHCH}_{3}\right), 1.53(\mathrm{~d}$, $\left.J_{\mathrm{HH}}=7.2 \mathrm{~Hz}, 3 / 2 \mathrm{H}, \mathrm{PCHCH}_{3}\right), 2.85\left(\mathrm{~m}, J_{\mathrm{HH}}=7.2 \mathrm{~Hz}, 1 \mathrm{H}\right.$, $\left.\mathrm{CH}\left(\mathrm{CH}_{3}\right)_{2}\right), 3.64\left(\mathrm{~d}, J_{\mathrm{HP}}=10.8 \mathrm{~Hz}, 3 \mathrm{H}, \mathrm{OCH}_{3}\right), 3.77\left(\mathrm{~d}, J_{\mathrm{HP}}=\right.$ $\left.10.8 \mathrm{~Hz}, 3 \mathrm{H}, \mathrm{OCH}_{3}\right), 4.64$ (s, 2H, $\left.\mathrm{OCH}_{2} \mathrm{CO}\right), 5.40$ (q, $J_{\mathrm{HH}}=7.2$ $\mathrm{Hz}, 1 \mathrm{H}, \mathrm{OCHP}), 6.82\left(\mathrm{~d}, J_{\mathrm{HH}}=7.8 \mathrm{~Hz}, 2 \mathrm{H}\right.$, phenyl-H), $7.09(\mathrm{~d}$, $J_{\mathrm{HH}}=7.8 \mathrm{~Hz}, 2 \mathrm{H}$, phenyl-H); ESI-MS m/z (\%) 684 (7), 683 $[2 \mathrm{M}+\mathrm{Na}]^{+}(22), 661(30), 660[2 \mathrm{M}+\mathrm{H}]^{+}(61), 353[\mathrm{M}+\mathrm{Na}]^{+}$ (17), $332(16), 331[\mathrm{M}+\mathrm{H}]^{+}$(100).

$O, O$-Dimethyl-1-(4-ethylphenoxyacetoxy)ethylphosphonate (22): yellow liquid; $R_{f}=0.55($ EtOAc $: n$-Hex $=4: 1) ;{ }^{1} \mathrm{H}$ $\mathrm{NMR}\left(\mathrm{CDCl}_{3} / \mathrm{TMS}\right) \delta 1.14\left(\mathrm{t}, J_{\mathrm{HH}}=7.8 \mathrm{~Hz}, 3 \mathrm{H}, \mathrm{CH}_{2} \mathrm{CH}_{3}\right)$, $1.43\left(\mathrm{~d}, J_{\mathrm{HH}}=7.2 \mathrm{~Hz}, 3 / 2 \mathrm{H}, \mathrm{PCHCH}_{3}\right), 1.46\left(\mathrm{~d}, J_{\mathrm{HH}}=7.2 \mathrm{~Hz}\right.$, $\left.3 / 2 \mathrm{H}, \mathrm{PCHCH}_{3}\right), 2.54$ (q, $\left.J_{\mathrm{HH}}=7.8 \mathrm{~Hz}, 2 \mathrm{H}, \mathrm{CH}_{2} \mathrm{CH}_{3}\right), 3.72(\mathrm{t}$, $\left.J_{\mathrm{HP}}=10.8 \mathrm{~Hz}, 6 \mathrm{H}, 2 \mathrm{OCH}_{3}\right), 4.62\left(\mathrm{~s}, 2 \mathrm{H}, \mathrm{OCH}_{2} \mathrm{CO}\right), 5.40$ (q, $\left.J_{\mathrm{HH}}=7.2 \mathrm{~Hz}, 1 \mathrm{H}, \mathrm{PCHO}\right), 6.76\left(\mathrm{~d}, J_{\mathrm{HH}}=8.4 \mathrm{~Hz}, 2 \mathrm{H}\right.$, phenyl-H), $7.09\left(\mathrm{~d}, J_{\mathrm{HH}}=8.4 \mathrm{~Hz}, 2 \mathrm{H}\right.$, phenyl-H); ESI-MS m/z (\%) 655 $[2 \mathrm{M}+\mathrm{Na}]^{+}(20), 634(22), 633[2 \mathrm{M}+\mathrm{H}]^{+}(68), 391(13), 390(65)$, $318(17), 317[\mathrm{M}+\mathrm{H}]^{+}(100)$.

Herbicidal activity inhibition assay. To measure the herbicidal activity $\left(O b s . \mathrm{pI}_{50}\right)$ against the seed of cucumber (Cucumis Sativus $L$.) of the analogues (1 22) were performed at least three times for verification. That is, the average value, depending on the growth length of roots, was calculated and the inhibitory ratio (\%) was accordingly measured. ${ }^{23,24}$

Molecular modeling. 3D-QSAR analyses were performed using Sybyl molecular modeling software (Ver. 8.0). ${ }^{25}$ All modeling operations were carried out under the same conditions $^{26}$ (alignments: the atom based fit $(\mathrm{AF})^{27} \&$ the field fit (FF); number of components: 1-5; grid: 1-3 $\AA$; field: the comparative molecular field analysis (CoMFA) fields: standard, indicator and $\mathrm{H}$-bond; the comparative molecular similarity indeces analysis (CoMSIA) fields: the electrostatic, the steric, the hydrophobic $(\log \mathrm{P})$, the H-bond acceptor and the H-bond donor). The Gästeiger- Hückel charge ${ }^{28}$ was used as the partial charge of a particular atom and the $\mathrm{AF}$ and $\mathrm{FF}$ alignment ${ }^{29}$ methods were respectively used as the spatial alignment of substrate molecules in three-dimensional space. AF alignment of the potent energy minimized substrate structures shown in Figure 1. Among the overall 22 data set compounds, 16 were the training set compounds used to drive of 3D-QSARs models. In order to the predictability, 6 of them were selected as the test set compounds.

PLS and scrambling analyses. The partial least squares (PLS) analysis method ${ }^{30}$ was used to analyze the correlation relation-

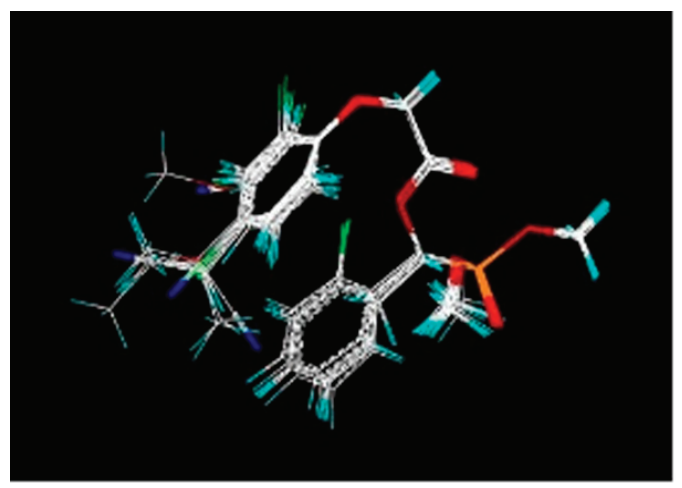

Figure 1. Alignment of the potential energy minimized $O, O$-dialkyl-1phenoxyacetoxy-1-methyl-phosphonate substrate structures according to a least-squares atom based fit. 
ships between the biological activity and the descriptors of the substrate molecule. Using this, the optimal 3D-QSARs models were induced and statistical values such as the predictability $\left(\mathrm{q}^{2} \& \mathrm{r}_{\text {ncv. }}^{2}\right)$ and the correlation coefficient $\left(\mathrm{r}^{2}\right.$ ncv. $)$ were obtained. To visually analyze the structural characteristics of the substrate molecules based on the optimized 3D-QSARs models, the CoMFA and CoMSIA field's properties were expressed as contour maps with in three-dimensional space. Contour maps (steve* coeff: favor: disfavor $=80: 20$ ) were generated by plotting the coefficients from the CoMFA and CoMSIA field. Also the progressive scrambling analysis ${ }^{31}$ was used to evaluate (maximum: 8 bins, minimum: 2 bins and critical point: 0.85 ) the dependence related to chance the correlation of the optimized CoMSIA models.

\section{Results and Discussion}

Optimized 3D-QSAR model. The observed herbicidal activity $\left(O b s . \mathrm{pI}_{50}\right)$ due to the $\mathrm{R}_{1} \sim \mathrm{R}_{4}$-substituents change on the $O$-phenyl ring of substrate molecules and the predicted herbicidal activity (Pred. $\mathrm{pI}_{50}$ ) from the CoMFA model I and the CoMSIA model I were summarized in Table 1. Among the substrate molecules, compound $2\left(O b s . p I_{50}=7.54\right)$ had the highest herbicidal activity and compound $\mathbf{1 0}\left(O b s \cdot \mathrm{pI}_{50}=3.24\right)$ showed the lowest herbicidal activity. The herbicidal activity difference between the two compounds was $\Delta O b s . \mathrm{pI}_{50}=4.30$. Especially, the

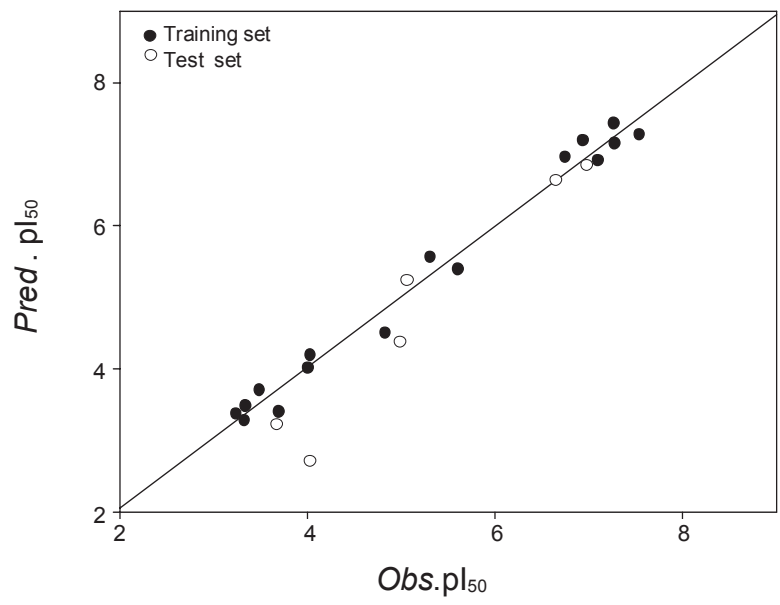

Figure 2. Relationships between observed activities (Obs.pI $\left.\mathrm{I}_{50}\right)$ against the cucumber and predicted activities (Pred.pI $\left.{ }_{50}\right)$ by the CoMSIA model I. (For the training set: Pred.pI $\mathrm{I}_{50}=0.985$ Obs.pI $\mathrm{I}_{50}+0.08, \mathrm{n}=16$, $\mathrm{S}=0.218, \mathrm{~F}=858.893, \mathrm{r}^{2}=0.984$ and $\left.\mathrm{q}^{2}=0.981\right)$.

observed herbicidal activities $\left(O b s . \mathrm{pI}_{50}\right)$ of the halogen atom substituted compounds $(\mathbf{1} \sim \mathbf{4}$ and $\mathbf{1 3} \sim \mathbf{1 6})$ were more high than those of the other substituents. Table 2 presents the statistical results of the optimal models in $3 \mathrm{D}-\mathrm{QSAR}$ models. These four models were selected from over 150 models that were calculated from conditions such as the CoMFA field and CoMSIA field

Table 1. Observed herbicidal activity $\left(\mathrm{Obs}\right.$. $\left.\mathrm{pI}_{50}\right)$ of substrate molecules against cucumber (Cucumis sativa) and predicted activity (Pred.pI $\left.{ }_{50}\right)$ by $3 \mathrm{D}-\mathrm{QSAR}$ models for the training set

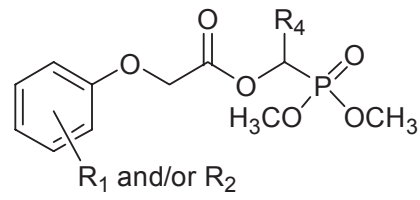

\begin{tabular}{|c|c|c|c|c|c|c|c|c|}
\hline \multirow{2}{*}{ No. } & \multicolumn{3}{|c|}{ Substituents(R) } & \multirow{2}{*}{$O b s . \mathrm{pI}_{50}$} & \multicolumn{2}{|c|}{ CoMFA model I } & \multicolumn{2}{|c|}{ CoMSIA model I } \\
\hline & $\mathrm{R}^{1}$ & $\mathrm{R}^{2}$ & $\mathrm{R}^{3}$ & & Pred.pI ${ }_{50}{ }^{a}$ & $\Delta \mathrm{pI}_{50}{ }^{b}$ & Pred.pI ${ }_{50}^{a}$ & $\Delta \mathrm{pI}_{50}{ }^{b}$ \\
\hline 1 & $2-\mathrm{F}$ & $4-\mathrm{Cl}$ & 2-ClPh & 7.27 & 7.43 & -0.16 & 7.43 & -0.16 \\
\hline 2 & $2-\mathrm{Cl}$ & $4-\mathrm{Br}$ & 2-ClPh & 7.54 & 7.24 & 0.30 & 7.27 & 0.27 \\
\hline 4 & $2-\mathrm{Cl}$ & $4-\mathrm{F}$ & 2-ClPh & 7.28 & 7.52 & -0.24 & 7.15 & 0.13 \\
\hline 6 & $\mathrm{H}$ & $3-\mathrm{CN}$ & 2-ClPh & 4.83 & 4.99 & -0.16 & 4.50 & 0.33 \\
\hline 8 & $\mathrm{H}$ & $4-\mathrm{OCH}_{3}$ & 2-ClPh & 5.31 & 5.46 & -0.15 & 5.56 & -0.25 \\
\hline 10 & $\mathrm{H}$ & 4- $\mathrm{CH}_{2} \mathrm{CH}_{2} \mathrm{CH}_{3}$ & 2-ClPh & 3.24 & 3.03 & 0.21 & 3.37 & -0.13 \\
\hline 11 & $\mathrm{H}$ & $4-\mathrm{CH}\left(\mathrm{CH}_{3}\right)_{2}$ & 2-ClPh & 3.70 & 3.57 & 0.13 & 3.40 & 0.30 \\
\hline 12 & $\mathrm{H}$ & $4-\mathrm{CH}_{2} \mathrm{CH}_{3}$ & 2-ClPh & 3.49 & 3.65 & -0.16 & 3.70 & -0.21 \\
\hline 13 & $2-\mathrm{F}$ & $4-\mathrm{Cl}$ & $\mathrm{CH}_{3}$ & 6.94 & 6.94 & 0.00 & 7.19 & -0.25 \\
\hline 14 & $2-\mathrm{Cl}$ & $4-\mathrm{Br}$ & $\mathrm{CH}_{3}$ & 7.10 & 6.95 & 0.15 & 6.91 & 0.19 \\
\hline 16 & $2-\mathrm{Cl}$ & $4-\mathrm{F}$ & $\mathrm{CH}_{3}$ & 6.75 & 6.77 & -0.02 & 6.96 & -0.21 \\
\hline 17 & $\mathrm{H}$ & $4-\mathrm{CN}$ & $\mathrm{CH}_{3}$ & 4.01 & 3.90 & 0.11 & 4.01 & 0.00 \\
\hline 18 & $\mathrm{H}$ & $3-\mathrm{CN}$ & $\mathrm{CH}_{3}$ & 4.03 & 4.03 & 0.00 & 4.19 & -0.16 \\
\hline 19 & $\mathrm{H}$ & $4-\mathrm{CH}_{2} \mathrm{CN}$ & $\mathrm{CH}_{3}$ & 3.33 & 3.50 & -0.17 & 3.28 & 0.05 \\
\hline 20 & $\mathrm{H}$ & $4-\mathrm{OCH}_{3}$ & $\mathrm{CH}_{3}$ & 5.61 & 5.22 & 0.39 & 5.39 & 0.22 \\
\hline 22 & $\mathrm{H}$ & $4-\mathrm{CH}_{2} \mathrm{CH}_{3}$ & $\mathrm{CH}_{3}$ & 3.34 & 3.56 & -0.22 & 3.48 & -0.14 \\
\hline
\end{tabular}

${ }^{a}$ Predicted values by the models, ${ }^{b}$ different between observed and predicted value. 
Table 2. Summary of the statistical parameters of 3D-QSAR models with two alignments

\begin{tabular}{|c|c|c|c|c|c|c|c|c|}
\hline \multirow{2}{*}{ Models No. } & \multirow{2}{*}{ Alignments } & \multicolumn{7}{|c|}{ PLS Analyses } \\
\hline & & $\operatorname{Grid}(\AA)$ & $\alpha^{a}$ & $\mathrm{NC}$ & $\mathrm{r}_{\mathrm{cv} .}^{2 b}$ & $\mathrm{r}_{\text {ncv. }}^{2}$ & $\mathrm{SE}_{\text {ncv. }}{ }^{d}$ & $\mathrm{~F}^{e}$ \\
\hline CoMFA I & $\mathrm{AF}$ & 2.0 & - & 4 & 0.535 & 0.986 & 0.229 & 198.794 \\
\hline CoMFA II & $\mathrm{FF}$ & 1.5 & - & 4 & 0.410 & 0.960 & 0.393 & 65.366 \\
\hline CoMSIA I ${ }^{f}$ & $\mathrm{AF}$ & 1.5 & 0.3 & 4 & 0.699 & 0.984 & 0.247 & 169.419 \\
\hline CoMSIA II & $\mathrm{FF}$ & 1.0 & 0.3 & 4 & 0.703 & 0.980 & 0.279 & 132.534 \\
\hline
\end{tabular}

Notes: AF: atom based fit; FF: field fit; NC: number of component; ${ }^{a}$ attenuation factor; ${ }^{b}$ cross- validated $\mathrm{r}^{2} ;{ }^{c}$ non-cross-validated $\mathrm{r}^{2} ;{ }^{d}$ standard error estimate; ${ }^{e}$ fraction of explained versus unexplained variance; ${ }^{f}$ optimized model.

Table 3. Summary of field contribution, Ave. and PRESS of 3D-QSAR models

\begin{tabular}{|c|c|c|c|c|c|c|c|c|}
\hline \multirow{2}{*}{ Model No. } & \multicolumn{4}{|c|}{ Field contribution $(\%)$} & \multicolumn{2}{|c|}{ Training set } & \multicolumn{2}{|c|}{ Test set } \\
\hline & $\mathrm{S}$ & $\mathrm{E}$ & Hy & HA & Ave. $^{b}$ & PRESS & Ave. ${ }^{b}$ & PRESS \\
\hline CoMFA I & 79.0 & 16.3 & 4.7 & - & 0.16 & 0.57 & 0.62 & 3.40 \\
\hline CoMFA II & 49.0 & 33.1 & 17.8 & - & 0.26 & 1.70 & 0.65 & 4.26 \\
\hline CoMSIA I $^{a}$ & 11.7 & 41.4 & 20.8 & 26.0 & 0.19 & 0.68 & 0.48 & 2.81 \\
\hline CoMSIA II & 11.2 & 61.3 & 27.5 & - & 0.18 & 0.72 & 0.64 & 4.59 \\
\hline
\end{tabular}

Notes: S: steric; E: electrostatic; Hy: hydrophobic; HA: H-bond Acceptor; Ave.: average residual; PRESS: Predictive residual sum of squares; ${ }^{a}$ optimized model; ${ }^{b}$ average residual of training set.

Table 4. Observed herbicidal activity $\left(\right.$ Obs.pI $\left.{ }_{50}\right)$ of substrate molecules against cucumber $($ Cucumis sativa $)$ and predicted activity $\left(\right.$ Pred.pI $\left.\mathrm{I}_{50}\right)$ by 3D-QSAR models for the test set

\begin{tabular}{|c|c|c|c|c|c|c|c|c|}
\hline \multirow{2}{*}{ No. } & \multicolumn{3}{|c|}{ Substituents(R) } & \multirow{2}{*}{ Obs.pI $\mathrm{I}_{50}$} & \multicolumn{2}{|c|}{ CoMFA I } & \multicolumn{2}{|c|}{ CoMSIA I $^{a}$} \\
\hline & $\mathrm{R}_{1}$ & $\mathrm{R}_{2}$ & $\mathrm{R}_{3}$ & & Pred.pI ${ }_{50}^{b}$ & $\Delta \mathrm{pI}_{50}^{c}$ & Pred.pI ${ }_{50}^{b}$ & $\Delta \mathrm{pI}_{50}{ }^{c}$ \\
\hline 3 & $2-\mathrm{Cl}$ & $3-\mathrm{Cl}$ & 2-ClPh & 6.95 & 5.92 & 1.03 & 6.83 & 0.12 \\
\hline 5 & $\mathrm{H}$ & $4-\mathrm{CN}$ & 2-ClPh & 5.00 & 5.18 & -0.18 & 4.33 & 0.67 \\
\hline 7 & $\mathrm{H}$ & $4-\mathrm{CH}_{2} \mathrm{CN}$ & 2-ClPh & 4.07 & 3.07 & 1.00 & 2.61 & 1.46 \\
\hline 9 & $\mathrm{H}$ & $3-\mathrm{OCH}_{3}$ & 2-ClPh & 5.06 & 5.11 & -0.05 & 5.20 & -0.14 \\
\hline 15 & $2-\mathrm{Cl}$ & $3-\mathrm{Cl}$ & $\mathrm{CH}_{3}$ & 6.68 & 5.61 & 1.07 & 6.60 & 0.08 \\
\hline 21 & $\mathrm{H}$ & 4- $\mathrm{CH}\left(\mathrm{CH}_{3}\right)_{2}$ & $\mathrm{CH}_{3}$ & 3.68 & 3.27 & 0.41 & 3.25 & 0.43 \\
\hline
\end{tabular}

${ }^{a}$ Optimized model; ${ }^{b}$ predicted values by the models; ${ }^{c}$ different between observed and predicted value.

Table 5. Model stability test for models by progressive scrambling

\begin{tabular}{|c|c|c|c|c|c|c|}
\hline \multirow{2}{*}{ No. } & \multicolumn{3}{|c|}{ CoMFA model I } & \multicolumn{3}{|c|}{ CoMSIA model I $^{a}$} \\
\hline & $\mathrm{q}^{2 b}$ & $\operatorname{cSDEP}^{c}$ & $\mathrm{~d}_{\mathrm{q}}^{2} / \mathrm{dr}^{2}{ }_{\mathrm{yy}}{ }^{d}$ & $\mathrm{q}^{2 b}$ & $\operatorname{cSDEP}^{c}$ & $\mathrm{~d}_{\mathrm{q}}{ }^{2} / \mathrm{dr}^{2} \mathrm{yy}^{\prime}{ }^{d}$ \\
\hline 2 & 0.309 & 1.486 & 0.345 & 0.481 & 1.290 & 0.780 \\
\hline 3 & 0.395 & 1.436 & 1.665 & 0.474 & 1.341 & 1.143 \\
\hline 4 & 0.426 & 1.463 & 1.085 & 0.503 & 1.356 & 0.830 \\
\hline 5 & 0.464 & 1.489 & 1.045 & 0.464 & 1.484 & 1.023 \\
\hline
\end{tabular}

${ }^{a}$ Optimized model; ${ }^{b} \mathrm{q}^{2}=1$-(sSDEP) $)^{2}$, predictivity of the models; ${ }^{c}$ calculated cross-validated standard error as function of correlation coefficient between the true values ( $\mathrm{y}$ ) of the dependent variables and the perturbed values ( $\mathrm{y}^{\prime}$ ) of the dependent variables; ${ }^{d}$ slope of $\mathrm{q}^{2}$ (cross-validated correlation coefficient from Sybyl) with respect correlation of the original dependent variables versus the perturbed dependent variables.

integrations, two alignments, the number of components and $\operatorname{grid}(\AA)$. In general, the statistical values of the CoMSIA models were more statistically satisfactory than those of the CoMFA models $\left(r_{\text {cv. }}^{2} \& r_{\text {ncv. }}^{2}\right.$ : CoMSIA model $\mathrm{I} \geq$ CoMSIA model II $\geq$ CoMFA model I $>$ CoMFA model II). The best model was CoMSIA model I $\left(\mathrm{r}_{\text {cv. }}^{2}\left(\right.\right.$ or $\left.\left.\mathrm{q}^{2}\right)=0.699 \& \mathrm{r}_{\text {ncv. }}^{2}=0.984\right)$ that were induced under the AF alignment condition. Figure 2 shows the correlation equation between the observed $O b s \cdot \mathrm{pI}_{50}$ and the predicted Pred.pI $\mathrm{I}_{50}$ values obtained from the CoMSIA model I (Pred.pI $I_{50}=0.985$ Obs.pI $I_{50}+0.08, \mathrm{n}=16, \mathrm{~S}=0.218, \mathrm{~F}=$ $\left.858.893, \mathrm{r}^{2}=0.984 \& \mathrm{q}^{2}=0.981\right)$.

Contribution ratio and predictability. The contribution ratio 


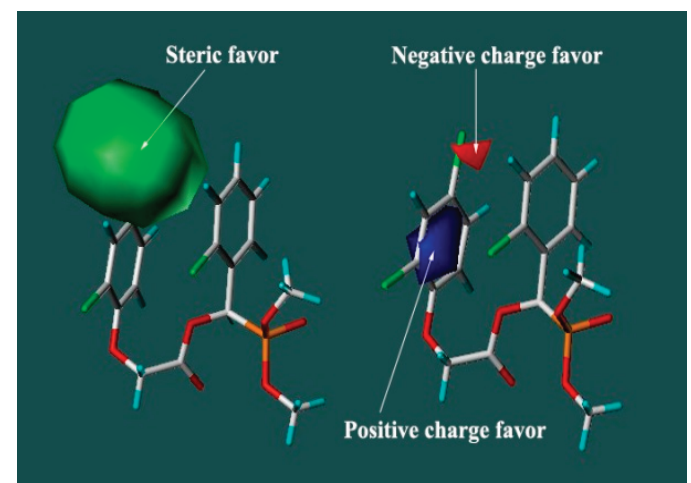

Figure 3. The contour map (stdev*coeff) of the CoMSIA model I for the steric field (left) and electrostatic field (right). The most active compound (2) is shown in capped sticks. (favor: $80 \%$ \& disfavor: $20 \%$ ).

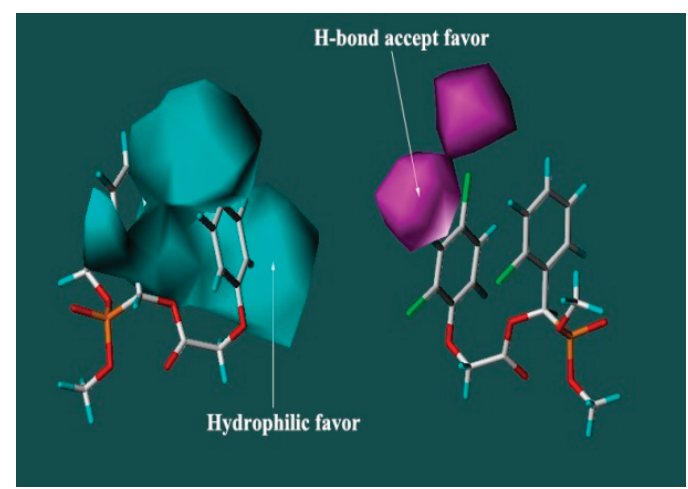

Figure 4. The CoMSIA model I contour map for the hydrophobic field and the H-bond accept field (stdev*coeff). The most active compound (2) is shown in capped sticks. (the hydrophobic favor and the H-bond accept favor : $80 \%$ \& the hydrophilic favor and the H-bond accept disfavor $20 \%$ ).

(\%), the average residual values (Ave.) and the PRESS values of the training set and test set compounds with 3D-QSAR models were summarized in Table 3 . The relative contribution ratios (\%) of the optimized CoMSIA model I were: the electrostatic field, 41.4; the H-bond acceptor field, 26.0; the hydrophobic field, 20.8 and the steric field, $11.7 \%$, respectively. Also, From the Ave. and the predictive residual sum of squares (PRESS) values of the training sets, it was once again verified that the optimized CoMSIA model I is a highly suitable model. Therefore, Table 4 shows the Obs.pI ${ }_{50}$ of the test set and the Pred.pI ${ }_{50}$ by the CoMFA model I and the CoMSIA model I as well as the two values' difference (Dev.). Compared to the CoMFA model I, the difference due to the CoMSIA model I was smaller.

Progressive scrambling. In Table 5, the three types of statistical data $\left(\mathrm{q}^{2}, \mathrm{cSDEP} \text {, and } \mathrm{d}_{\mathrm{q}}^{2^{2}} / \mathrm{dr}_{\mathrm{yy}}^{2}\right)^{32}$ were summarized related to the model's dependence and obtained from the progressive scrambling analysis of the CoMFA model I and the CoMSIA model I. Values of the $\mathrm{q}^{2}$ and cSDEP are the predictivity of the models and the calculated cross-validated standard error, respectively. And the susceptibility of the model can be gauged by the slope to perturbation $\left(\mathrm{d}_{\mathrm{q}}{ }^{2} / \mathrm{dr}^{2} \mathrm{yy}\right.$ ) of $\mathrm{q}^{2}$ (as originally determined using SAMPLS) with respect to the correlation of the original biological activity versus the scrambled biological activity. As a result, the CoMSIA model I showed better predictability at the component 4 with $\mathrm{q}^{2}>0.5$. Also, The perturbation value $\left(\mathrm{d}_{\mathrm{q}}{ }^{2} / \mathrm{dr}^{2}{ }_{\mathrm{yy}}\right)$ of the CoMFA model I was 1.085 and that of the CoMSIA model I was 0.830 . These values were satisfied the condition ${ }^{33}$ that optimized models without chance correlation should have a gradient value $\left(\mathrm{d}_{\mathrm{q}}^{2} / \mathrm{dr}^{2}{ }^{\prime} y^{\prime}=0.8 \sim 1.2\right)$ at component 4 . Therefore, it explains that the optimized CoMSIA model I is independent of chance correlation and very appropriate.

CoMSIA contour maps. The steric and the electrostatic field CoMSIA contour maps on the most active compound 2 (Obs. $\mathrm{pI}_{50}=7.54$ ) is shown in Figure 3. The steric favor groups (the green polyhedra) are in the $\mathrm{R}_{1}$ and $\mathrm{R}_{2}$-phenyl substituents. As the sizes of the $R_{1}$ and $R_{2}$-phenyl substituents raises the herbicidal activities are increased. In the case of $\mathrm{R}_{1}$ and $\mathrm{R}_{2}$-substituents (para-position) on the $O$-phenyl ring, the transformation of a steric bulky group ${ }^{21}$ will be increased the herbicidal activity. The positive charge favored area on the $O$-phenyl ring is represented in the blue contours and the negative charge favored area on the para-position on the $O$-phenyl ring shows in the red contours. As more positively charged substituents are introduced on the $O$-phenyl ring, the herbicidal activity is increased. Also, it can be observed that on the contour maps (Fig. 4) of the hydrophobic $(\log \mathrm{P})$ field and the $\mathrm{H}$-bond acceptor field. Specifically, it can be predicted that as more hydrophilic substituents (cyan polyhedral region) are introduced to the $\mathrm{R}_{1}, \mathrm{R}_{2}$-position on the $O$-phenyl ring and the $\mathrm{R}_{4}$-substituent there will be increased in the herbicidal activity. According to the results of the contour map in the H-bond acceptor field on the right side, the herbicidal activity was predicted increase by the H-bond acceptor favor substituents (purple polyhedral region) as oxygen atom and nitrogen atom not adjacent to a hydrogen atom on the $\mathrm{C}_{3}$ and $\mathrm{C}_{4}$-atoms of the $O$-phenyl ring.

\section{Conclusions}

A series of $O, O$-dialkyl-1-phenoxyacetoxy-1-methylphosphonate analogues (1 22) as a new class of potent inhibitors of pyruvate dehydrogenase were synthesized. And 3D-QSARs (CoMFA and CoMSIA) models between the structures of the analogues and their pre-emergency herbicidal activity against the seed of cucumber (Cucumus Sativa L.) were derived and discussed quantitatively. The optimized CoMSIA model I that showed the best statistical values in 3D-QSAR models, had the predictability value and the correlation coefficient vlaue of $\mathrm{r}_{\text {cv. }}^{2}\left(\right.$ or q $\left.^{2}\right)=0.699$ and $\mathrm{r}_{\text {ncv. }}^{2}=0.984$, respectively. The contribution percentages (\%) of the optimized CoMSIA model I were: the electrostatic field, 41.4; the H-bond acceptor field, 26.0; the hydrophobic field, 20.8 and the steric field, $11.7 \%$. According to the progressive scrambling analyses results, the optimized CoMSIA model I $\left(\mathrm{q}^{2}=0.503 \& \mathrm{~d}_{\mathrm{q}}{ }^{2} / \mathrm{dr}^{2} \mathrm{yy}^{\prime}=0.830\right)$ was a highly suitable model that did not depend on chance correlation. It is predicted that in the electrostatic field contour maps of the CoMSIA model I, the more positively charged substituent on the $O$-phenyl ring and the more negatively charged substituent on the para-position on the $O$-phenyl ring, the herbicidal activity will be increase. 
Acknowledgments. This work was supported by National Research Foundation (NRF) grant (No. 2010-0062913) funded by the Korea Government (MEST).

\section{References}

1. Yoshiba, Y.; Kiyosue, T.; Nakashima, K.; Yamaguchi-Shinozaki, K.; Shinozaki, K. Plant Cell Physiol.1997, 38, 1095-1102.

2. Hare, P. D.; Cress, W. A. Plant Growth Regul. 1997, 21, 79-102.

3. Hare, P. D.; Cress, W. A. V.; Staden, J. Plant Growth Regul. 2003, 39, 41-50.

4. Schwacke, R.; Grallath, S.; Breitkreuz, K. E.; Stransky, E.; Stransky, H.; Frommer, W. B.; Rentsch, D. Plant Cell 1999, 11, 377391.

5. Giuseppe, F.; Samuele, G. I.; Lukasz, B.; Davide, P.; Pawel, K. J. Agric. Food Chem. 2007, 55, 4340-4347.

6. Gohda, K.; Kimura, Y.; Mori, I.; Ohta, D.; Kikuchi, T. Biochim. Biophys. Acta 1998, 1385, 107-114.

7. Hawkes, T. R.; Cox, J. M.; Barnes, N. J.; Beautement, K.; Edwards, L. S.; Kipps, M. R.; Langford, M. P.; Lewis, T.; Ridley, S. M.; Thomas, P. G. Proceedings of the Brighton Crop Protection Conference 1993, 6, 739-744.

8. Gutowski, J. A.; Lienhard, G. E. J. Biol. Chem. 1976, 251, $2863-$ 2866.

9. Dobritzsch, D.; Konig, S.; Schneider, G.; Lu, G. J. Biol. Chem. 1998, 273, 20196-20204.

10. Kluger, R.; Pike, D. C. J. Am. Chem. Soc. 1977, 99, 4504-4057.

11. Mahler, H. R.; Cordes, E. H. In Biological Chemistry, 2nd ed.; Harper and Row: New York, 1971; p 519.

12. Koike, M.; Reed, L. J.; Caroll, W. R. J. Biol. Chem. 1963, 238, 30-39.

13. Alvarez, F. J.; Ermer, J.; Hubner, G.; Schellenberger, A.; Schowen, R. L. J. Am. Chem. Soc. 1991, 113, 8402-8409.

14. Kern, D.; Kern, G.; Neef, H.; Tittmann, K.; Killenberg-Jabs, M.; Schneider, C. W.; Hubner, G. Science 1997, 275, 67-70.

15. Baillie, A. C.; Wright, K.; Wright, B. J.; Earnshaw, C. G. Pestic.
Biochem. Physiol. 1988, 30, 103-112.

16. He, H. W.; Wang, J.; Liu, Z. J. J. Org. Chem. 2001, 21, 878-883.

17. Wang, T.; He, H. W.; Yuan, J. L. J. Appl. Chem. 2003, 20, 613-617.

18. He, H. W.; Wang, T.; Yuan, Z. L. J. Organometallic Chem. 2005, 690, 2608-2613.

19. Lee, E. T.; Kim, S. D. Kor. J. Appl. Microbiol. Biotechnol. 2000, $28,334-340$.

20. He, H. W.; Chen, T.; Li, Y. J. Pesticide Sci. 2007, 32, 42-44.

21. Kubinyi, H. In $3 D$ QSAR in Drug Design, Theory, Methods and Applications; Ludwigshafen: ESCOM Leiden, Germany, 1993.

22. Sung, N. D.; Jang, S. C.; Hwang, T. Y. Korean J. Pestic. Sci. 2007, $11,72-81$.

23. Chen, T.; Shen, P.; Li, Y.; He, H. W. Phos. Sul. Silic. 2006, 181, 2135-2145.

24. Peng, H.; Wang, T.; Xie, P.; Chen, T.; He, H. W.; Wan, J. J. Agric. Food Chem. 2007, 55, 1871-1880.

25. Tripos, Molecular modeling and QSAR software on CD-Rom (Ver. 8.0); Tripos Associates, Inc.: 1699 S. Hanley Road, Suite 303, St. Louis, MO., U.S.A.

26. Soung, M. G.; Lee, Y. J.; Sung, N. D. Bull. Korean Chem. Soc. 2009, 30, 613-617.

27. Marshall, G. R.; Barry, C. D.; Bosshard, H. E.; Dammkoehler, R. A.; Dunn, D. A. In computer-assisted drug design: The conformational parameter in drug design; active analog approach; Olsen, E. C., Christoffersen, R. E., Eds.; American Chemical Society: Washington, D. C., 1979; p 205.

28. Purcell, W. P.; Singer, J. A. J. Chem. Eng. Data 1967, 122, $235-$ 246.

29. Clark, M.; Cramer, R. D., III.; Jones, D. M.; Patterson, D. E.; Simeroth, P. E. Tetrahedron Comput. Methodol. 1990, 3, 47-59.

30. Cramer, R. D.; Bunce, J. D.; Patterson, D. E. Quant. Struct. Act. Relat. 1988, 7, 18-25.

31. Clark, R. D.; Fox, P. C. J. Computer-Aided molecular Design 2004, 18, 563-576.

32. Juan, A. A. S.; Cho, S. J. J. Mol. Model. 2007, 13, 601-610.

33. Ashek, A.; Cho, S. J. Bioorg. \& Med. Chem. 2005, 14, 1474-1482. 